\title{
Congenital Pseudoarthrosis of the Tibia: A Case Report
}

\author{
Raj kumar meena ${ }^{1}$, Arun Kumar G C ${ }^{1}$, S Nongthon Singh ${ }^{2}$, Sanjib Nepram ${ }^{4}$ \\ ${ }^{I}$ (PGT, Department of Orthopaedics, Regional Institute of Medical Sciences, Manipur, India) \\ ${ }_{2}^{2}$ (Professor, Department of Orthopaedics, Regional Institute of Medical Sciences, Manipur, India) \\ ${ }^{3}$ (Associate Professor and Head, Department of Plastic Surgery, Regional Institute of Medical Sciences, \\ Manipur, India)
}

\begin{abstract}
Congenital pseudoarthrosis of the tibia is a rare disease with reported incidence ranging from 1:140000 to $1: 250000$ neonates. It is characterized by segmental osseous weakness resulting in deformities of the bone and spontaneous fractures which progresses to a tibial non - union. The etiology is unknown. In 50\% of the cases it is associated with neurofibromatosis . The pathologic process of the disorder is the growth of abnormal fibromatosis like tissue either within the periosteum (dysplastic type) or both.It is one of the most challenging problem in paediatric orthopaedics. Various methods of treatment are modifications of illizarov's technique, vascularised bone grafting, bone grafting with intramedullary nail fixation. We report a case of 11 year old boy having congenital pseudoarthrosis of left tibia with no prior history of any surgical treatment . During surgery, sclerotic ends of both fragment of pseudoarthrosis excised, reduction and fixation done using intra medullary nail with bone grafting. Six months later radiograph showed bony union with acceptable functional out come and no refracture. It is shown that intramedullary fixation with bone grafting is a good choice for treatment of congenital pseudoarthosis of tibia .
\end{abstract}

Keywords: congenital, fixation, intramedullary, pseudoarthrosis, tibia

\section{Case Report}

A 11 years old boy presented to Regional Institute of Medical Sciences, Orthopaedics department with complaint of deformity at left lower leg since birth and difficulty in walking .There was no family history of such deformity and no associated neurofibromatosis. After reviewing the radiographs and clinical profile, a diagnosis of congenital pseudoarthrosis of tibia(CPT), boyd's type 4 was made (fig 1 and 2) and he was planned for surgical correction. Pseudoarthrosis was locater at the junction of middle and distal third of tibia . During surgery pseudoarthrosis was exposed, the sclerotic surfaces of the fragments were excised, intramedullary nail fixation and cancellous bone grafing done from iliac crest. An image intensifier was used to confirm the desired position of fragments and the implant .

Pieces of cancellous bone harvested from iliac crest were placed in between the fragments and around the excised pseudoarthrosis site.Wound infection developed at the osteotomy site which was managed with appropriate antibiotics after culture and sensitivity testing. There was some skin loss at operated site for which skin grafing was done.Two months after surgery, a custom made shoe with $3 \mathrm{~cm}$ elevation to compensate for the difference in the length of the left lower extremity was made and walking was started with support under supervision. At six month follow up, patient was able to walk without support and there was no pain at operated site and radiograph showed bony union (fig 3 and 4).

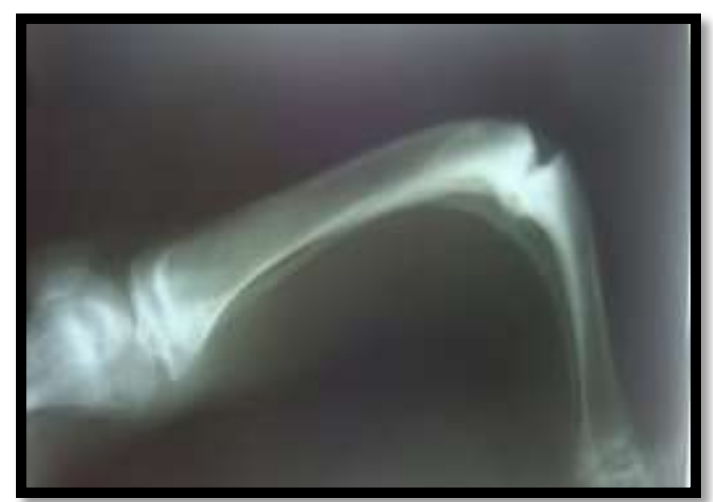

Figure 1

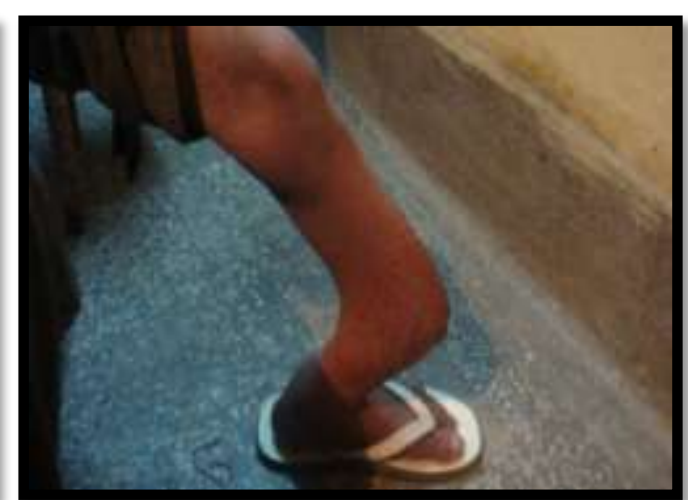

Figure 2 


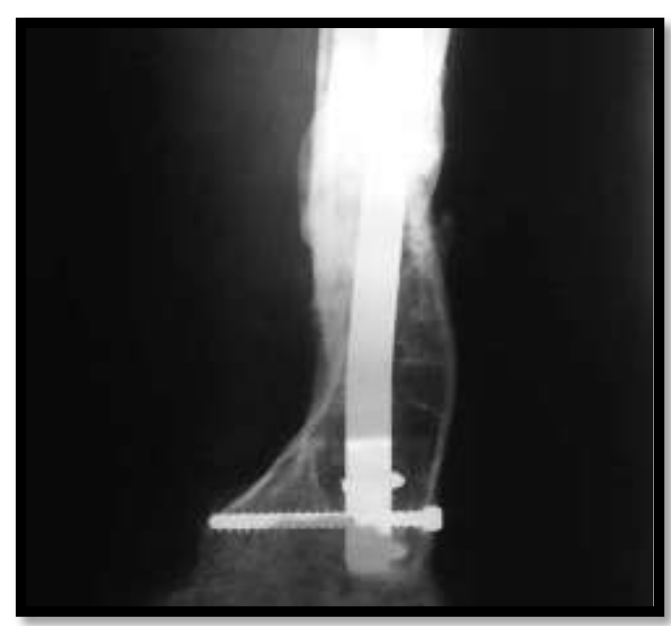

Figure 3

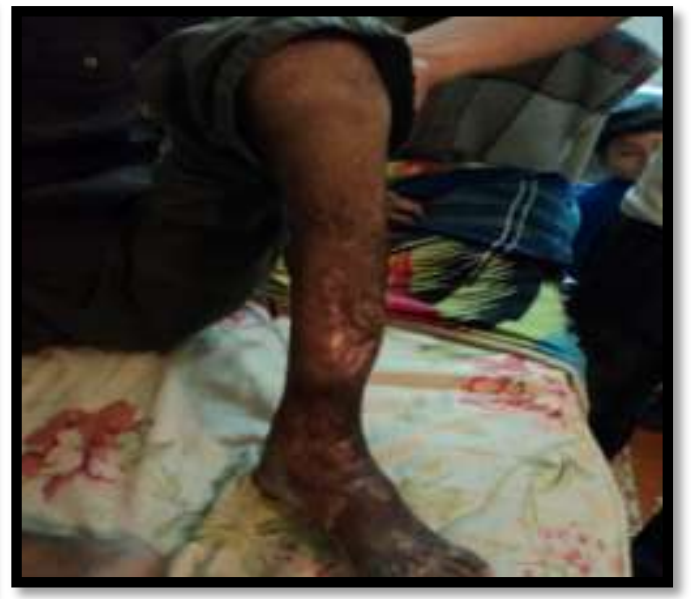

Figure 4

\section{Discussion}

Congenital pseudoarthrosis of tibia(CPT) is a rare disease characterized by segmental osseous weakness resulting in anterolateral angulation of the bone and spontaneous fractures which progresses to a tibial non union $^{1,2}$.It is a very rare entity with a reported incidence of $1: 140,000$ to $1: 250,000$ neonates ${ }^{3}$. The congenital osseous dysplasia in the distal third of the tibia with fibroblastic tissue which disrupts callus formation and leads to a tibial non-union .

The etiology is unknown. In upto $50 \%$ of the cases, it is associated with a neurofibromatosis . In others, heriditory and mechanical factors are implicated. Various classification system have been proposed (Anderson, boyd, Crawford). Usually the disease becomes evident in a child within two years of life but may be undetected up to the age of 12 years ${ }^{1,2}$.

CPT has been classified into 6 different types according to boyd's classification ${ }^{4}$. Type 1 is described as anterior bowing with a defect in the tibia. Type 2 is dysplatic lesion with anterolaterl bowing and hourglass constriction. This is often associated with neurofibromatosis. Type 3 is a cystic deformity with bone cysts at the junction of the middle and lower third of tibia . Type 4 is described as originating in a sclerotic segment of the tibia without any narrowing or fractures. Type 5 is dysplastic fibula and Type 6 is a intraosseous nerofibroma or schwannoma with or without pseudoarthrosis . Our case was classified in type 4 .

Intramedullary stabilization, free vascularised fibula graft and illizarov's external fixation are among the most frequently used methods of treatment . In addition bone morphogenetic protein has shown promising results. It is often resistant to standard treatment and refractures are common. CPT continues to be a challenge to orthopaedic surgeons ${ }^{5}$. The reported union rates for different techniques are $90 \%$ for bone grafting with intra medullary nail fixation , $81 \%$ for vascularised bone grafting and $71 \%$ for illizarov's technique.The pathologic process of the disorder is growth of abnormal fibromatosis like tissue either within periosteum ( dysplastic type )or its coexistence of both (mixed type $)^{6}$. Complete excision of the abnormal tissue is necessary to treat the problem, evidenced by visualizing normal bleeding and marrow cavity. Resection of the abnormal tissue followed by fragmant approximation, intramedullary nail fixation and cancellous bone grafting resulted in bony union. There was wound dehiscence at osteotomy site which required skin grafting. The intramedullary nail provided stability and alignment and resulted in bony union with good functional outcome .

\section{Conclusion}

Congenital pseudoarthrosis of tibia is a very rare entity and causes great difficulty in treatment . In our case, intramedullary nail fixation with bone grafting provided good result. Intramedullary nail fixation preserves local circulation, produces adequate compression resulting in bone union .

\section{References}

[1] 1.H andreas ,S gundula ,H benita , W G rolf, American Journal of Roentgenology, 177 (2001) 1025.

[2] 2. AD delgado-martinez, E Crodrigez-merchan, B olsen , Int Orthop, 20 (1996) 192.

[3] $3 \mathrm{~F}$ hefti ,G bollini , P dungl , J Pediatr Orthop B, 9 (2000) 11.

[4] 4 HB Boyd, Pathology and natural history of congenital pseudarthrosis of tibia. Clin Orthop 1982; 166: 5-13

[5] 5. K sauli ,V vesa ,S willy, Acta Orthop Belgica, 74 (2008) 132

[6] 6.S Wientroub ,F Grill . Congenital pseudarthrosis of the tibia: part 1. European Pediatric Orthopaedic Society multicenter study of congenital pseudoarthrosis. J Pediatr Orthop B 2000;9:1-2. 